\title{
Is cardiac CT a reproducible alternative for cardiac MR in adult patients with a systemic right ventricle?
}

\author{
M. M. Winter • S. Romeih • B. J. Bouma • M. Groenink • \\ N. A. Blom • A. M. Spijkerboer • B. J. M. Mulder
}

Published online: 31 July 2012

(C) Springer Media / Bohn Stafleu van Loghum 2012

\begin{abstract}
Objective $20 \%$ of patients with a systemic RV are pacemaker dependent, and unsuitable to undergo cardiac magnetic resonance (CMR). Multidetector row computed tomography (MDCT) could provide a reproducible alternative to CMR in these patients. The aim of this study was to compare variability of MDCT with CMR.

Methods Thirty-five patients with systemic RV underwent either MDCT $(n=15)$ or CMR $(n=20)$. Systemic RV volumes and ejection fraction were obtained, and intra- and interobserver variability for both modalities were assessed and compared.

Results We found the intra- and interobserver variability of volumes and function measurements of the systemic RV obtained with MDCT to be higher compared with those obtained with CMR. However, these differences in variability were not significant, the only exception being the interobserver variability of systemic RV stroke volume.
\end{abstract}

M. M. Winter · S. Romeih • B. J. Bouma • M. Groenink •

B. J. M. Mulder $(\square)$

Department of Cardiology, Academic Medical Center,

B2-240, Meibergdreef 9,

1105 Amsterdam, the Netherlands

e-mail: b.j.mulder@amc.uva.nl

URL: www.concor.net

M. M. Winter · S. Romeih • B. J. M. Mulder Interuniversity Cardiology Institute of the Netherlands, Utrecht, the Netherlands

S. Romeih $\cdot$ M. Groenink $\cdot$ A. M. Spijkerboer

Department of Radiology, Academic Medical Center,

Amsterdam, the Netherlands

N. A. Blom

Department of Paediatrics, Academic Medical Center,

Amsterdam, the Netherlands
Conclusions MDCT provides a reproducible alternative to $\mathrm{CMR}$ for volumes and function assessment in patients with a systemic RV.

Keywords Adult congenital heart disease - Transposition of the great arteries - Multidetector row computed tomography · Cardiovascular magnetic resonance imaging

\section{Background}

Patients with a complete transposition of the great arteries (TGA) who had undergone an atrial switch operation in the past and patients with a congenitally corrected transposition of the great arteries (ccTGA) have a morphological right ventricle (RV) supporting the systemic circulation. Due to improvements in palliative cardiac surgery early in life, the number of adult patients with a systemic RV has increased dramatically over the past few decades [1]. Although longterm outcome in these patients is unknown, morbidity is worrisome, with tricuspid valve regurgitation, arrhythmias, and RV dysfunction being the main constituents $[2,3]$.

Reliable assessment of systemic right ventricular volumes and function is important for clinical decision making, to follow-up therapeutic intervention, and to properly execute clinical research [4, 5]. Currently, cardiac magnetic resonance (CMR) is considered the gold standard for accurate and reproducible systemic right ventricular volumes and function assessment [5, 6]. However, $20 \%$ of patients with a systemic RV are pacemaker or implantable cardioverterdefibrillator (ICD) dependent [7, 8], and an increasing number of patients with a failing systemic RV benefit from cardiac resynchronisation therapy [9]. As most intracardiac devices are considered to be CMR incompatible, these patients are unsuitable to undergo CMR. Multidetector row 
computed tomography (MDCT) may provide a reliable alternative to CMR in these patients.

Although the accuracy of MDCT measurements of cardiac volumes and function is relatively well documented, no studies have been performed on the reproducibility of measurements $[10,11]$. Therefore, the objective of our study was to evaluate intra- and interobserver variability of the right ventricular volumes and function measurements by MDCT, in comparison with CMR, in patients with a systemic RV.

\section{Methods}

Patient characteristics

A cross-sectional prospective study was performed among 35 consecutive patients with a systemic RV, 23 patients with an atrially switched TGA, and 12 with a ccTGA. All patients had RV volumes and function evaluation either by CMR ( $n=20$; mean age: $35 \pm 12$ years) in patients without, or by MDCT ( $n=15$; mean age: $32 \pm 8$ years) in patients with a pacemaker or ICD.

The Human Research Committees of all participating institutions approved the study protocol, and the study protocol conforms with the ethical guidelines of the 1975 Declaration of Helsinki. Written informed consent was obtained from all patients prior to participation in the study.

\section{Image acquisition}

For MDCT image acquisition, contrast-enhanced retrospective ECG-gated MDCT was performed using Philips Brilliance-64 Computed Tomography scanner. All scans were obtained during breath-hold at the end of inspiration. Patients received $90 \mathrm{ml}$ of a contrast medium $(70 \mathrm{ml}$ at a flow rate of $5.0 \mathrm{ml} / \mathrm{s}$, followed by a $20 \mathrm{ml}$ at a flow rate of $3.5 \mathrm{ml} / \mathrm{s}$, and a $40 \mathrm{ml}$ bolus of saline at a flow rate of $3.5 \mathrm{ml} / \mathrm{s}$ ) containing $300 \mathrm{mg}$ of iodine (Iomeron 300, Bracco Imaging SpA, Milan, Italy). No $\beta$-blocker preparation was used. The scan was automatically commenced after contrast detection in the systemic RV. The contrast detection threshold was set at 150 Hounsfield units. The rotation time was $0.4 \mathrm{~s}$, and the pitch factor was 0.2 . The tube current was $600 \mathrm{MA}$, the tube voltage was $120 \mathrm{kV}$, and the effective radiation dose per scan was around $14 \mathrm{mSv}$. Two-millimetre thick contiguous slices were reconstructed in $512 \times 512$ matrix using a $100 \mathrm{~mm}$ field of view. The whole heart was covered within $60-80$ slices per cardiac phase. Data in steps of $10 \%$ of R-R interval (ranging from $0 \%$ to $90 \%$ for each investigation) were obtained using a segmental reconstruction algorithm. From these axial images, multi-planar reformations in the short-axis orientation, with a slice-thickness of $6 \mathrm{~mm}$, without slice gap, were done. This resulted in 12 to 15 short-axis slices, which were used for functional analysis.

CMR was performed using 1.5 Tesla scanner (Siemens Avanto, Erlangen, Germany), two- and four-chamber and short-axis views covering both ventricles from the base of the heart to the apex were acquired using a retrospective ECG-gated steady-state free precession (SSFP) sequence during breath holding at expiration. Short-axis view consisted of 12 to 15 contiguous slices. Scan parameters were: repetition time $=3.2-3.8 \mathrm{~ms}$; echo time $=1.6-1.9 \mathrm{~ms}$; flip angle $=50-70^{\circ}$; slice thickness $=6 \mathrm{~mm}$ without slice gap; matrix $=160 \times 256$; field-of-view $=350-400 \mathrm{~mm}$. Temporal resolution was approximately $25 \mathrm{~ms}$. All the data were stored in DICOM format and transferred to a PC workstation running a MASS program.

Image analysis

For MDCT and CMR image analysis we used the MASS Analytical Software System (Medis, Leiden, the Netherlands). Cine loops were used to choose end diastole and end systole. End diastole was defined as the phase with the largest right ventricular (and left ventricular (LV)) volume and end systole as the phase with the smallest right ventricular (and LV) volume. The slices at the base of the heart were considered to be in the ventricle if the blood was at least half surrounded by ventricular myocardium. It was only possible to optimise differentiation between the ventricles and atria and vessels in the basal slices using fourchamber and two-chamber views simultaneously with shortaxis views in the CMR group. Trabeculations and papillary muscles were considered part of the ventricular cavity [5]. The sums of the traced contours in end diastole and end systole were used to calculate the end-diastolic and endsystolic volume using a disc summation technique. Enddiastolic and end-systolic volumes were used to calculate stroke volume and ejection fraction. Stroke volume was defined as end-diastolic volume-end-systolic volume, and ejection fraction as [(end-diastolic volume-end-systolic volume)/end-diastolic volume] $\times 100 \%$. All ventricular volumes were indexed for body surface area according to the Mosteller formula: $(\sqrt{ }$ height $(\mathrm{cm}) \times$ weight $(\mathrm{kg}) / 3600)$.

Contours were traced in total 3 times by 2 independent observers (MW, SR) The first observer analysed all scans twice, with a minimal interval of 2 weeks between the first and second scan analysis, and blinded to the previous results. The second observer analysed the scans once, blinded to the results of the first observer.

\section{Statistics}

For statistical analyses, SPSS 16.0 (SPSS Inc., Chicago, Illinois) for Windows was used. $\mathrm{P}$ values $<0.05$ were 
considered statistically significant. The descriptive data are presented as mean with standard deviation if normally distributed, or as median with range as appropriate. Intra- and interobserver measurement variability was determined from the mean values and the differences between the 2 measurements, and visualised with the methods and plots as described by Bland and Altman. The coefficient of variability (CV) was calculated as the standard deviation of the difference of the paired measurements divided by the mean of the average of the paired measurements, and expressed as a percentage. The statistical comparison of any differences in reproducibility of MDCT and CMR measurements was assessed with an extension of the Bland-Altman methods. Therefore, a log transformation of the squared differences between the two measurements was performed. If the squared difference was 0 , we replaced the value by the next smallest value multiplied by 0.5 , before $\log$ transformation. A two-tailed unpaired $t$-test of the logged squared differences of MDCT versus CMR was performed thereafter [12].

\section{Results}

\section{Patient characteristics}

A total of 35 adult patients ( $66 \%$ male, mean age $33.6 \pm$ 10.7 years) with a systemic RV were included in the study, 23 patients with an atrially switched TGA, and 12 patients with a ccTGA.

CMR was performed in 20 patients, whereas 15 patients underwent MDCT due to implantation of pacemaker or ICD (14 patients with pacemakers, and 1 patient with an ICD). There were no statistically significant differences in age, type of TGA, and NYHA functional class between patients who underwent CMR and those who underwent MDCT. All CMR and MDCT scans were undertaken without complications. Patient characteristics are summarised in Table 1.

Systemic right ventricular volumes and function assessment

We found no statistically significant differences in intraobserver variability of end-diastolic volume, end-systolic volume, stroke volume and ejection fraction between measurements obtained by CMR, compared with MDCT. Moreover, we found no statistically significant differences in interobserver variability of end-diastolic volume, endsystolic volume, and ejection fraction between measurements obtained by CMR, compared with MDCT. However, CMR had a superior interobserver variability for stroke volume measurements compared with MDCT (12\% variability with CMR vs. $32 \%$ variability with MDCT; $p<0.01$ ), Fig. 1. These differences were statistically non-significant,
Table 1 Baseline characteristics

\begin{tabular}{lllll}
\hline Characteristics & $\begin{array}{l}\text { All patients } \\
(n=35)\end{array}$ & $\begin{array}{l}\text { CMR } \\
(n=15)\end{array}$ & $\begin{array}{l}\text { MDCT } \\
(n=20)\end{array}$ & P value \\
\hline Age (years) & $33.6 \pm 10.7$ & $34.8 \pm 12.5$ & $31.9 \pm 8.0$ & NS \\
Male & $23(66 \%)$ & $16(80 \%)$ & $7(47 \%)$ & 0.05 \\
BSA $\left(\mathrm{m}^{2}\right)$ & $1.9 \pm 0.04$ & $1.8 \pm 0.2$ & $1.9 \pm 0.3$ & $\mathrm{NS}$ \\
Heart rate (bpm) & $71 \pm 2$ & $71 \pm 16$ & $70 \pm 10$ & $\mathrm{NS}$ \\
NYHA class I & $77 \%$ & $75 \%$ & $80 \%$ & $\mathrm{NS}$ \\
NYHA class II & $14 \%$ & $20 \%$ & $7 \%$ & $\mathrm{NS}$ \\
NYHA class III & $9 \%$ & $5 \%$ & $13 \%$ & $\mathrm{NS}$ \\
NYHA class VI & $0 \%$ & $0 \%$ & $0 \%$ & $\mathrm{NS}$ \\
Atrially switched TGA & $23(66 \%)$ & $13(65 \%)$ & $10(67 \%)$ & $\mathrm{NS}$ \\
\hline
\end{tabular}

$B S A$ body surface area; NS not significant; NYHA New York Heart Association; TGA transposition of the great arteries

except for the interobserver variability for stroke volume measurements. However, the coefficient of variability was higher for all measurements performed with MDCT, except for the interobserver variability of end-systolic volumes ( $13 \%$ with CMR vs. $12 \%$ with MDCT; $p=n s)$. Intra- and interobserver variability data are summarised in Table 2.

\section{Discussion}

In the current study, we have shown for the first time that volumes and function measurement with MDCT is equally reproducible compared with assessment with CMR in patients with a systemic RV, and therefore provides an alternative for those patients who are unsuitable to undergo CMR.

In patients with normal cardiac anatomy, MDCT is already considered to be a reliable alternative for CMR for biventricular volumes and function measurements [10, 13]. However, the feasibility of routine use of MDCT in patients with a systemic RV cannot simply be extrapolated from these data, as the morphology of the systemic RV differs substantially from the subpulmonary RV. The complex geometric shape of the systemic RV, its extensive trabeculations and poor acoustic windows, make standard geometric assumptions impossible, and function assessment challenging $[14,15]$. Subsequently, quantitative assessment of the systemic RV with frequently used diagnostic modalities, such as echocardiography, is difficult $[16,17]$. MDCT, similar to CMR, has the ability to provide any desired imaging plane and does not rely on the geometric assumptions to calculate the right ventricular volume. However, its role in patients with a systemic RV had not yet been established.

The establishment of MDCT as a reproducible alternative for CMR is important, as $20 \%$ of patients with a systemic $\mathrm{RV}$ are pacemaker dependent, and an increasing number of 
Fig. 1 Bland-Altman plots depicting the intra- and interobserver variability between multidetector row computed tomography and cardiac magnetic resonance. Bland-Altman plots demonstrating the intraobserver (left side), and interobserver (right side) variability of right ventricular a) end-diastolic volume, b) end-systolic volume, c) stroke volume, and d) ejection fraction. On the $\mathrm{X}$-axis the mean value of both measurements, and on the Y-axis the difference between measurements. $\boldsymbol{\Delta}$ represents measurements performed with MDCT, - represents the mean of the differences between MDCT measurements, * represents measurements performed with CMR, - represents the mean of the differences between CMR measurements
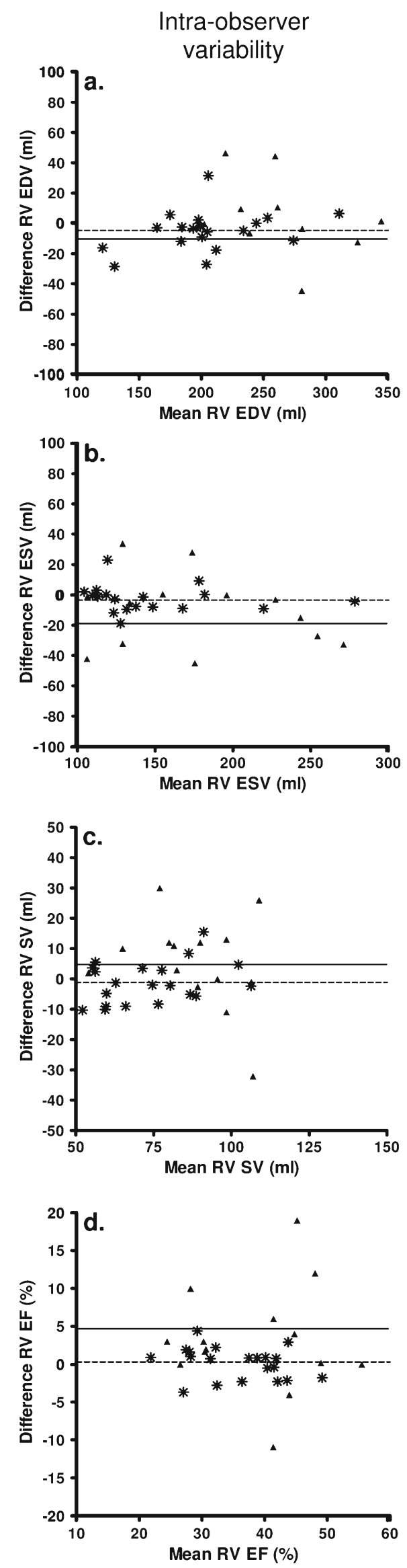

Inter-observer

variability
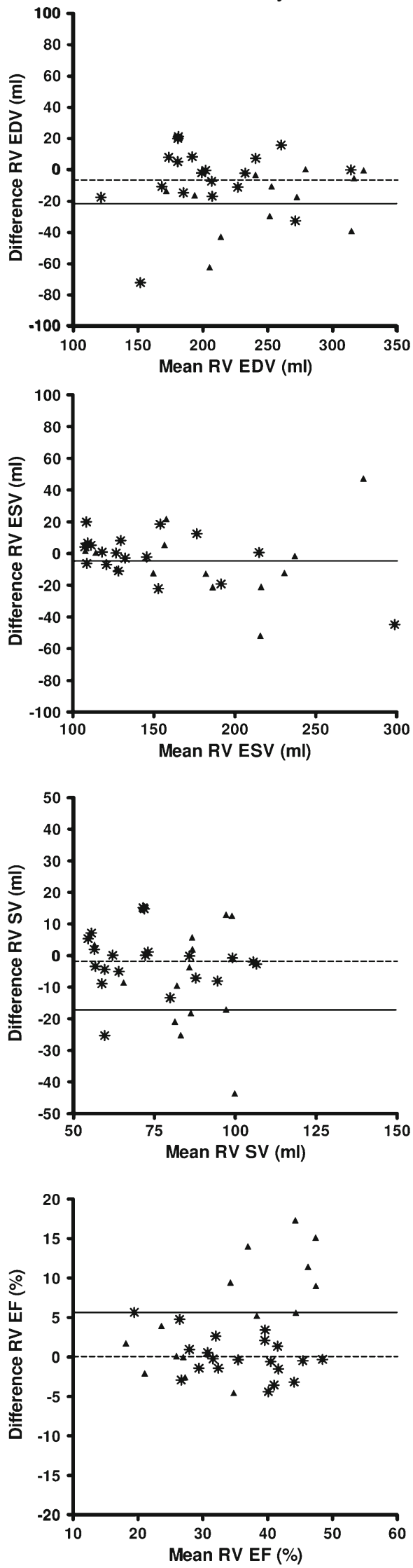
Table 2 Intra- and interobserver variability of measurements
$C V$ cardiac volume, $E D V$ enddiastolic volume, $E F$ ejection fraction, $E S V$ end-systolic volume, $N S$ not significant, $S V$ stroke volume

\begin{tabular}{|c|c|c|c|c|c|c|c|}
\hline \multirow[t]{2}{*}{ Parameter } & \multicolumn{3}{|c|}{ CMR $(n=20)$} & \multicolumn{3}{|c|}{$\operatorname{MDCT}(n=15)$} & \multirow[t]{2}{*}{ P-value } \\
\hline & Average & Difference & $\mathrm{CV}$ & Average & Difference & $\mathrm{CV}$ & \\
\hline \multicolumn{8}{|c|}{ Intraobserver variability } \\
\hline EDV (ml) & 212 & $-5 \pm 13$ & $6 \%$ & 294 & $-11 \pm 36$ & $12 \%$ & NS \\
\hline $\mathrm{ESV}(\mathrm{ml})$ & 139 & $-4 \pm 9$ & $7 \%$ & 200 & $-19 \pm 35$ & $18 \%$ & NS \\
\hline $\mathrm{SV}(\mathrm{ml})$ & 74 & $-1 \pm 7$ & $9 \%$ & 96 & $5 \pm 15$ & $16 \%$ & NS \\
\hline $\mathrm{EF}(\%)$ & 36 & $0.1 \pm 2$ & $6 \%$ & 35 & $5 \pm 9$ & $25 \%$ & NS \\
\hline \multicolumn{8}{|c|}{ Interobserver variability } \\
\hline $\mathrm{EDV}(\mathrm{ml})$ & 213 & $-7 \pm 21$ & $10 \%$ & 277 & $-22 \pm 34$ & $12 \%$ & NS \\
\hline ESV (ml) & 139 & $-5 \pm 18$ & $13 \%$ & 189 & $-5 \pm 22$ & $12 \%$ & NS \\
\hline $\mathrm{SV}(\mathrm{ml})$ & 74 & $-2 \pm 9$ & $12 \%$ & 89 & $-17 \pm 29$ & $32 \%$ & $<0.01$ \\
\hline $\mathrm{EF}(\%)$ & 36 & $-0.1 \pm 3$ & $8 \%$ & 35 & $-6 \pm 7$ & $20 \%$ & NS \\
\hline
\end{tabular}

patients are receiving cardiac resynchronisation therapy or ICDs [18]. Although data on CMR compatibility and safety of intracardiac devices remain limited and controversial, most intracardiac devices are currently considered to be CMR incompatible [19]. One study reports encouraging results on device safety when scanning patients with certain devices, if the right precautions are taken [20]. However, others have described a variety of mechanisms by which CMR could affect pacemaker and ICD function. The magnetic forces could attract and displace the pacemakers and ICDs [21], and could lead to 'reed switch activation' in sporadic cases [22]. Moreover, radiofrequency energy could cause heating of the intracardiac leads [23]. In summary, whether scanning patients with pacemakers and ICD is contraindicated remains disputable, as contraindications are predominantly theoretical, and clinical data are limited [22]. To obtain valid and accurate information on CMR compatibility and safety of intracardiac devices further research is warranted.

There are several restrictions that should be taken into account before MDCT is performed. Firstly, we found remarkable differences in reproducibility between MDCT and CMR, although they were not statistically significant. These differences are most likely due to the differences in image acquisition and image analysis between the 2 modalities. In MDCT temporal resolution remains limited in comparison with CMR, making MDCT more sensitive to cardiac motion and making the definition of end-systolic and end-diastolic time points less precise [24]. Using beta blocker medication to lower a patient's heart rate partially overcomes this problem, but is not desirable as this could change functional parameters. On the other hand, MDCT provides an excellent spatial resolution which, in combination with the administered contrast, enhances differentiation between blood and myocardium [25]. The lower reproducibility of MDCT parameters could also be due to differences in image analysis between MDCT and CMR. Although the protocol we used to draw contours was the same in the CMR group as in the MDCT group, the analytical software could provide us with 4and 2-chamber views simultaneously with the short-axis view in the CMR group but not in the MDCT group. This made differentiation between ventricles, atria and vessels in the basal slices challenging in the MDCT group.

Another important difference with CMR is patients' exposure to radiation and contrast agents during MDCT. Although the effective radiation dose per scan was around $14 \mathrm{mSv}$ in our study, effective radiation doses of up to $32 \mathrm{mSv}$ per scan have been reported [26]. The possible impact of this large quantity of radiation should not be taken lightly. Einstein et al. and Hurwitz et al. have reported that MDCT-derived coronary angiography, with an effective radiation dose ranging from 12 to $32 \mathrm{mSv}$, causes a significant increase in risk of both lung and breast cancer, especially in younger and female patients [27]. There are strategies by which radiation dose can be reduced, without reducing image quality to an unacceptable level; patients should only be scanned when they have a stable sinus rhythm, tube voltage can be lowered to 100 or $80 \mathrm{kV}$ in the small patients or the children, ECG-controlled tube current modulation can be used, and the scan volume should be accurately specified prior to scanning [26, 28]. Beside radiation, the administered contrast agent imposes a risk factor for patients undergoing MDCT. The risk of contrast-induced nephropathy is significant, especially in patients with risk factors, such as pre-existing renal function impairment or diabetes mellitus [29]. The risk of contrast-induced nephropathy can be reduced by prophylactic pre-hydration, but proper risk assessment of 
all patients prior to MDCT remains of key importance [30]. However, reticence and thorough patient selection remain key to avoid any unnecessary exposure to radiation or contrast agents.

As with most studies on MDCT or CMR in patients with congenital heart diseases, our study is limited by a relatively small number of patients. Moreover, we compared two different groups of patients: those who underwent CMR and those who underwent MDCT. However, we found no differences in characteristics between patients who underwent CMR, compared with those who underwent MDCT, except for sex distribution. All patients who underwent MDCT were unsuitable to undergo CMR due to the presence of intracardiac devices. We could have performed MDCT in patients without intracardiac devices to overcome this limitation, but chose not to unnecessarily expose these young patients to radiation and contrast agents.

\section{Conclusions}

Multidetector row computed tomography provides a reproducible alternative to cardiac magnetic resonance for ventricular volumes and function assessment in patients with a systemic right ventricle, although larger variability between measurements should be taken into account. Patient selection should be restrictive to avoid unnecessary exposure to radiation and contrast agents.

Acknowledgements This study was supported by an unrestricted educational grant from Novartis Pharma B.V.

Competing interests The authors declare that they have no competing interests.

Financial disclosures This study was supported by an unrestricted educational grant from Novartis Pharma B.V.

\section{References}

1. Marelli AJ, Mackie AS, Ionescu-Ittu R, et al. Congenital heart disease in the general population: changing prevalence and age distribution. Circulation. 2007;115:163-72.

2. Engelfriet P, Boersma E, Oechslin E, et al. The spectrum of adult congenital heart disease in Europe: morbidity and mortality in a 5 year follow-up period. The Euro Heart Survey on adult congenital heart disease. Eur Heart J. 2005;26:2325-33.

3. Warnes CA. Transposition of the great arteries. Circulation. 2006;114:2699-709.

4. Deanfield J, Thaulow E, Warnes C, et al. Management of grown up congenital heart disease. Eur Heart J. 2003;24:1035-84.

5. Winter MM, Bernink FJ, Groenink M, et al. Evaluating the systemic right ventricle by CMR: the importance of consistent and reproducible delineation of the cavity. J Cardiovasc Magn Reson. 2008;10:40.
6. Grothues F, Moon JC, Bellenger NG, et al. Interstudy reproducibility of right ventricular volumes, function, and mass with cardiovascular magnetic resonance. Am Heart J. 2004; 147:218-23.

7. Gelatt M, Hamilton RM, McCrindle BW, et al. Arrhythmia and mortality after the Mustard procedure: a 30-year single-center experience. J Am Coll Cardiol. 1997;29:194-201.

8. Lundstrom U, Bull C, Wyse RK, et al. The natural and "unnatural" history of congenitally corrected transposition. Am J Cardiol. 1990;65:1222-9.

9. Diller GP, Okonko D, Uebing A, et al. Cardiac resynchronization therapy for adult congenital heart disease patients with a systemic right ventricle: analysis of feasibility and review of early experience. Europace. 2006;8(4):267-72.

10. Dogan H, Kroft LJ, Bax JJ, et al. MDCT assessment of right ventricular systolic function. AJR Am J Roentgenol. 2006;186: S366-70.

11. Lembcke A, Dohmen PM, Dewey M, et al. Multislice computed tomography for preoperative evaluation of right ventricular volumes and function: comparison with magnetic resonance imaging. Ann Thorac Surg. 2005;79:1344-51.

12. Papavassiliu T, Kuhl HP, Schroder M, et al. Effect of endocardial trabeculae on left ventricular measurements and measurement reproducibility at cardiovascular MR imaging. Radiology. 2005;236:57-64.

13. Guo YK, Gao HL, Zhang XC, et al. Accuracy and reproducibility of assessing right ventricular function with 64-section multidetector row CT: Comparison with magnetic resonance imaging. Int J Cardiol. 2010;139:254-62.

14. Tulevski II, Romkes H, Dodge-Khatami A, et al. Quantitative assessment of the pressure and volume overloaded right ventricle: imaging is a real challenge. Int J Cardiovasc Imaging. 2002;18:41-51.

15. Wilson NJ, Neutze JM, Rutland MD, et al. Transthoracic echocardiography for right ventricular function late after the Mustard operation. Am Heart J. 1996;131:360-7.

16. Dodge-Khatami A, Tulevski II, Bennink GB, et al. Comparable systemic ventricular function in healthy adults and patients with unoperated congenitally corrected transposition using MRI dobutamine stress testing. Ann Thorac Surg. 2002;73:1759-64.

17. Tulevski II, Lee PL, Groenink M, et al. Dobutamine-induced increase of right ventricular contractility without increased stroke volume in adolescent patients with transposition of the great arteries: evaluation with magnetic resonance imaging. Int J Card Imaging. 2000;16:471-8.

18. Abraham WT, Fisher WG, Smith AL, et al. Cardiac resynchronization in chronic heart failure. N Engl J Med. 2002;346:1845-53.

19. Naehle CP, Kreuz J, Strach K, et al. Safety, feasibility, and diagnostic value of cardiac magnetic resonance imaging in patients with cardiac pacemakers and implantable cardioverters/defibrillators at 1.5 T. Am Heart J. 2011;161:1096-105.

20. Nazarian S, Roguin A, Zviman MM, et al. Clinical utility and safety of a protocol for noncardiac and cardiac magnetic resonance imaging of patients with permanent pacemakers and implantable-cardioverter defibrillators at 1.5 tesla. Circulation. 2006;114:1277-84.

21. Roguin A, Zviman MM, Meininger GR, et al. Modern pacemaker and implantable cardioverter/defibrillator systems can be magnetic resonance imaging safe: in vitro and in vivo assessment of safety and function at $1.5 \mathrm{~T}$. Circulation. 2004;110:475-82.

22. Shinbane JS, Colletti PM, Shellock FG. MR in patients with pacemakers and ICDs: Defining the issues. J Cardiovasc Magn Reson. 2007;9:5-13.

23. Achenbach S, Moshage W, Diem B, et al. Effects of magnetic resonance imaging on cardiac pacemakers and electrodes. Am Heart J. 1997;134:467-73. 
24. Nieman K, Rensing BJ, van Geuns RJ, et al. Non-invasive coronary angiography with multislice spiral computed tomography: impact of heart rate. Heart. 2002;88:470-4.

25. Boll DT, Bossert AS, Aschoff AJ, et al. Synergy of MDCT and cine MRI for the evaluation of cardiac motility. AJR Am J Roentgenol. 2006;186:S379-86.

26. Mayo JR, Leipsic JA. Radiation dose in cardiac CT. AJR Am J Roentgenol. 2009;192:646-53.

27. Einstein AJ, Henzlova MJ, Rajagopalan S. Estimating risk of cancer associated with radiation exposure from 64-slice computed tomography coronary angiography. JAMA. 2007;298:317-23.
28. Hausleiter J, Meyer T, Hadamitzky M, et al. Radiation dose estimates from cardiac multislice computed tomography in daily practice: impact of different scanning protocols on effective dose estimates. Circulation. 2006;113:130510 .

29. Manske CL, Sprafka JM, Strony JT, et al. Contrast nephropathy in azotemic diabetic patients undergoing coronary angiography. Am J Med. 1990;89:615-20.

30. Tepel M, van Der GM, Schwarzfeld C, et al. Prevention of radiographic-contrast-agent-induced reductions in renal function by acetylcysteine. N Engl J Med. 2000;343:180-4. 